\title{
Bloqueo de la rama lingual del nervio gloso- faríngeo en pacientes pediátricos sometidos a adenotonsilectomía: estudio prospectivo y de corte transversal
}

\author{
Lingual branch of glossopharyngeal nerve block in pediatric \\ patients undergoing adenotonsillectomy: prospective and \\ cross-sectional clinical trial
}

Adrián Vera ${ }^{1}$, César Little ${ }^{1}$, Mula Haro ${ }^{1}$

\begin{abstract}
Objective: To determine the efficacy of lingual branch of glossopharyngeal nerve block in postoperative management of pain in pediatric patients undergoing adenotonsillectomy. Methods: Prospective and cross-sectional clinical trial. The sample was 105 patients between 3 and 8 years old scheduled for adenotonsillectomy, who performed lingual branch of glossopharyngeal nerve block by anterior intraoral approach with bupivacaine $0.5 \%$ + adrenaline 1:200,000. Postoperative pain using CHEOPS scale, complications, need for postoperative analgesics, recovery time using modified Aldrete scale and time for oral tolerance was assessed. Results: We studied 101 patients classified into three groups according to the level of postoperative pain divided into mild (88.1\%), moderate $(7.9 \%)$ and severe $(4 \%)$, with means and DS for discharged time of $50.13 \pm$ $13.912 \mathrm{~min}, 63.75 \pm 10.607 \mathrm{~min}$ and $86.25 \pm 7.5 \mathrm{~min}$; first oral intake $3.08 \pm$ $0.829 \mathrm{~h}, 4 \pm 0 \mathrm{~h}$ and $4 \pm 0 \mathrm{~h}$; and, first post-discharge pain reference of $18.3 \pm$ $7.414 \mathrm{~h}, 5.75 \pm 0.866 \mathrm{~h}$ and $4.5 \pm 1 \mathrm{~h}$ respectively. Analgesic reinforcement was needed in $11.9 \%$ of the cases. There were not complications. Conclusions: Lingual branch of glossopharyngeal nerve block is a useful and effective tool in the management of pain and anesthetic recovery of pediatric patients undergoing adenotonsillectomy.
\end{abstract}

Key words:

Nerve Blocks, glossopharyngeal nerve, analgesia, pediatrics tonsillectomy

Anestesiólogos adjuntos al Servicio de Anestesiología Hospital General del Este Dr. Domingo Luciani, Caracas, Venezuela.

Fecha de recepción: 25 de junio de 2018

Fecha de aceptación: 22 de noviembre de 2018

ORCID

https://orcid.org/0000-0003-0473-8099

Correspondencia:

Email: 


\section{RESUMEN}

Objetivo: Determinar la eficacia del bloqueo de la rama lingual del nervio glosofaríngeo en el manejo postoperatorio del dolor en pacientes pediátricos para adenotonsilectomía. Métodos: Estudio con diseño prospectivo y de corte transversal. La muestra consistió en 105 pacientes con edades comprendidas entre los 3 y 8 años de edad programados para adenotonsilectomía, a los que se realizó bloqueo de la rama lingual del nervio glosofaríngeo abordaje intraoral anterior con bupivacaína 0,5\% + adrenalina 1:200.000. Se estudió el dolor postoperatorio mediante escala CHEOPS, complicaciones, necesidad de analgésicos postoperatorio, tiempo de recuperación mediante escala de Aldrete modificado y tiempo para tolerancia oral. Resultados: Se estudiaron 101 pacientes en tres grupos según el grado de dolor postoperatorio divididos en leve $(88,1 \%)$, moderado $(7,9 \%)$ y severo (4\%), con medias y DS para tiempo de alta de 50,13 $\pm 13,912 \mathrm{~min}, 63,75 \pm 10,607$ min y $86,25 \pm 7,5$ min; primera ingesta oral 3,08 $\pm 0,829 \mathrm{~h}, 4 \pm 0 \mathrm{~h}$ y $4 \pm 0 \mathrm{~h}$; $\mathrm{y}$, primera referencia de dolor posterior al alta de $18,3 \pm 7,414$ h, $5,75 \pm 0,886$ h y $4,5 \pm 1$ h respectivamente. Se ameritó refuerzo analgésico en el $11,9 \%$ de los casos. No se evidenciaron complicaciones. Conclusiones: El bloqueo de la rama lingual del nervio glosofaríngeo es una herramienta útil y eficaz en el manejo del dolor y recuperación anestésica de pacientes pediátricos sometidos a adenotonsilectomía.

\section{Introducción}

a adenotonsilectomía (ADT) es un procedimiento quirúrgico muy común, siendo el segundo en la - población pediátrica y alcanzando los 530.000 procedimientos anuales en menores de 15 años en los Estados Unidos. Es utilizado como método definitivo para el tratamiento de la tonsilitis a repetición, los desórdenes respiratorios del sueño y la apnea obstructiva del sueño (AOS)[1].

La principal causa de morbilidad posterior a la ADT es el dolor orofaríngeo, con pico máximo los primeros días y que puede prolongarse hasta por dos semanas. A causa del dolor, es observado que disminuye la ingesta oral y causa deshidratación, prolonga el tiempo de hospitalización e incluso puede llevar al reingreso hospitalario[2].

Los opioides son la base del tratamiento del dolor postoperatorio (DPO), pero presentan efectos adversos como depresión respiratoria, sedación, náuseas y vómitos. La búsqueda de una pronta recuperación de los pacientes, con retorno a la nutrición enteral y actividades normales, hacen que el uso de los opioides esté limitado. Los analgésicos antiinflamatorios no esteroideos (AINEs) pueden aumentar el sangrado y traer problemas gastrointestinales[3].

Los bloqueos de nervio periférico se presentan

\section{Palabras clave:}

Bloqueo nervioso, nervio glosofaríngeo, analgesia, pediátricos, admigdalectomía como una opción con pocos efectos adversos para el manejo del DPO. En la búsqueda bibliográfica sobre los bloqueos nerviosos en pacientes pediátricos $y$, aquellos utilizados para ADT, se hace mención a la infiltración peritonsilar y al bloqueo del nervio glosofaríngeo (NGF), procedimiento sencillo el cual brinda analgesia hasta por 72 horas, no obstante, destacan la falta de ensayos clínicos suficientes para apoyar el uso de esta última en la población pediátrica[4].

Desde que la anestesia regional ha demostrado ser una estrategia válida para mejorar los resultados en el manejo del dolor postoperatorio en la población adulta, es importante examinar más a fondo el efecto de estas técnicas en pacientes pediátricos, por lo que nos planteamos la siguiente pregunta: ¿Será el bloqueo de la rama lingual (BRL) del NGF con bupivacaína 0,5\% + adrenalina 1:200.000, eficaz para la analgesia postoperatoria en pacientes pediátricos sometidos a ADT?

Como objetivo principal del estudio se planteó determinar la eficacia del bloqueo de la rama lingual del nervio glosofaríngeo en el manejo postoperatorio del dolor en pacientes pediátricos sometidos a adenotonsilectomía. Dentro de los objetivos secundarios a investigar se propuso evaluar el dolor postoperatorio, cuantificar los requerimientos analgésicos en el postoperatorio inmediato, medir el tiempo de recuperación 
anestésica, registrar el tiempo de la primera ingesta oral en el postoperatorio y describir las complicaciones postoperatorias asociadas a la técnica.

\section{Pacientes y Métodos}

Se diseñó un estudio experimental, prospectivo y de corte transversal, el cual se llevó a cabo en un hospital terciario. Después de la aprobación por parte del comité de ética de la institución y obtener el consentimiento informado de los padres o representantes por escrito, 105 pacientes con edades comprendidas entre 3 y 8 años y estado físico ASA I y || programados para ADT con técnica de disección fría o caliente con electrocauterio, fueron incluidos en el estudio. Fueron excluidos aquellos individuos con deformidades craneofaciales, cirugía previa de cara, cuello o cavidad oral, AOS, contraindicación para la aplicación de técnicas regionales (coagulopatías, infección en el sitio de punción), alergia a algunos de los fármacos utilizados, más de dos intentos para la intubación orotraqueal y tiempo quirúrgico mayor de 90 minutos.

Diez minutos antes de ingresar a quirófano, los pacientes recibieron premedicación intravenosa (IV) con midazolam 0,05 mg/kg, ranitidina $1 \mathrm{mg} / \mathrm{kg}$, metoclopramida $0,2 \mathrm{mg} / \mathrm{kg}$, dexametasona $0,2 \mathrm{mg} / \mathrm{kg}$ y metamizol $10 \mathrm{mg} / \mathrm{kg}$.

Una vez que el paciente ingresara al quirófano, se les colocó monitorización tipo ASA II estándar con cardioscopio, oximetría de pulso, presión arterial no invasiva (PANI), capnografía, temperatura y analizador de gases inspirados y espirados. La inducción anestésica se realizó con los siguientes fármacos IV: fentanilo $2 \mu \mathrm{g} / \mathrm{kg}$, lidocaína $1 \% 1 \mathrm{mg} / \mathrm{kg}$ y propofol $1 \% 2 \mathrm{mg} / \mathrm{kg}$.

Se llevó a cabo la laringoscopia directa e intubación orotraqueal, con tubo endotraqueal convencional con manguito inflable adecuado al paciente, se conectó a la máquina de anestesia y se mantuvo con modo volumen controlado con los parámetros correspondientes a edad y peso del individuo. El mantenimiento de la anestesia fue realizado con $\mathrm{O}_{2}$ y aire a un volumen total de 2 litros con fracción inspirada de $\mathrm{O}_{2}$ de 0,6 y sevoflurane a una concentración alveolar mínima de 1 medido por analizador de gases.

Previo a la incisión quirúrgica, se preparó una mezcla con bupivacaína al 0,5\% + adrenalina 1:200.000 y se aplicó la técnica con una aguja 25G. Para la realización de la misma, el médico encargado de realizar el bloqueo introdujo la hoja del laringoscopio y desplazó la lengua en sentido contrario hasta visualizar la base del arco palatogloso anterior, punto donde se intro- dujo la aguja en $90^{\circ}$ con una profundidad 1,5 mm, se aspiró para evitar la inyección intravascular inadvertida y se administró la mezcla con un volumen de 0,05 $\mathrm{ml} / \mathrm{kg}$ por lado.

Al culminar el acto quirúrgico, se procedió a realizar aspiración de secreciones orales y, posterior a la extubación en primer plano anestésico, el paciente fue trasladado al área de recuperación. El individuo permaneció en esta área hasta que reunió criterios de alta según la escala de Aldrete modificado y se valoró el DPO según la The Children's Hospital of Eastern Ontario Pain Scale (CHEOPS) cada 15 minutos hasta su egreso. En base al DPO que presentaron los individuos medido por la CHEOPS, estos se clasificaron en 3 grupos de la siguiente manera: $\leq$ 4 puntos dolor leve, 5 - 10 dolor moderado y $\geq 10$ puntos dolor severo; por lo que los análisis de los datos se realizaron conforme a las características de cada grupo.

Si se observaba que el paciente presentaba una puntuación mayor a 5 puntos según la CHEOPS se le administraba morfina $40 \mu \mathrm{g} / \mathrm{kg}$ y se registró en el instrumento de recolección de datos. El tiempo hasta la primera ingesta oral y la primera referencia de DPO también fue registrado. La recolección de los datos fue realizada por un observador independiente el cual no estaba vinculado con el estudio.

En caso de que el paciente presentara alguna reacción adversa a los medicamentos estudiados, a la técnica empleada o complicaciones relacionadas a la cirugía, se realizaron las intervenciones necesarias para el tratamiento de las mismas y se registraron en el instrumento de recolección de datos. Así mismo, se registró la presencia de complicaciones asociadas a la técnica de bloqueo (náuseas y vómitos postoperatorios, hemorragias, parálisis de nervios faríngeos, debilidad o paresia de la lengua, abolición del reflejo tusígeno, inyección intravascular y hematomas).

El cálculo del tamaño de la muestra se realizó con la fórmula para muestras con población infinita o desconocida: $P=0,5, q=0,5, Z$ para $95 \%$ de confianza, $i=$ error máximo permitido para un intervalo de confianza de $95 \%$ y un error de $8 \%$; para un total de 98 pacientes. Se obtuvo las estadísticas descriptivas (valor mínimo, valor máximo, media y desviación típica) de las variables cuantitativas y porcentajes en las variables cualitativas. Así mismo se realizaron contrastes de hipótesis de la varianza en las variables cuantitativas y prueba chi cuadrado en las variables cualitativas, los contrastes se hicieron con un nivel de significación de 5\%; se utilizó el programa SPSS (paquete estadístico aplicado a las ciencias sociales) versión 23. 


\section{Resultados}

Se evaluó una muestra de 105 niños, de los cuales fueron excluidos 4 pacientes: dos por diagnóstico de AOS, uno por presentar dificultades para la intubación y otro por prolongación del tiempo quirúrgico (Figura 1).

En relación a las variables demográficas: la media de edad fue 5,14 \pm 1,8 años, el peso de 20,3 \pm 7,37 kilogramos $(\mathrm{kg})$ y la talla $110,76 \pm 14,55$ centímetros (cm). Se observó que 66 pacientes $(66,33 \%)$ pertenecieron al género masculino y $35(33,67 \%)$ al género femenino, y la distribución con respecto al estado físico según ASA, 85 fueron ASA I (84,2\%) y 16 ASA II $(15,8 \%)$. En cuanto a las características de la cirugía, para el tiempo quirúrgico se halló una media de 44,83 $\pm 13,7$ minutos, con una distribución de 14 pacientes intervenidos con técnica fría (13,9\%) y 87 con técnica caliente $(86,1 \%)$ (Tabla 1$)$.

En referencia a la evaluación del dolor, el 88,1\% de los pacientes presentaron puntuaciones según la CHEOPS menor o igual a 4 puntos, 7,9\% de la muestra tuvo puntaje entre 5 - 10 puntos y el $4 \%$ más 10 puntos, con diferencia estadísticamente significativa

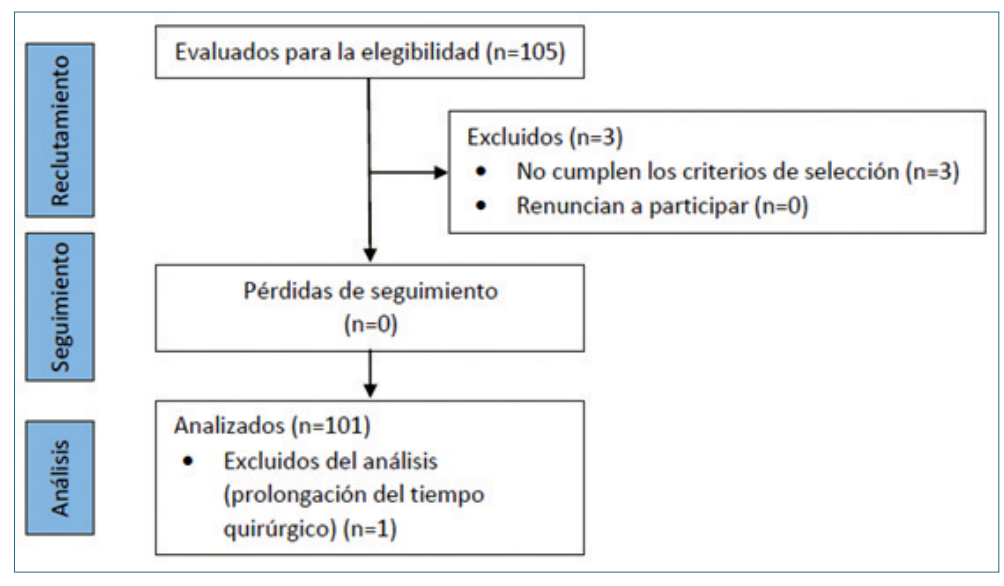

Figura 1. CONSORT.

\begin{tabular}{lcccc}
\multicolumn{5}{c}{ Tabla 1. Característica de la muestra } \\
\hline Característica & $\mathbf{n}$ & media & DE & $\%$ \\
Edad (años) & 101 & 5,14 & 1,8 & - \\
Sexo & & & - & 66,33 \\
Masculino & 67 & - & - & 33,67 \\
Femenino & 34 & - & 7,37 & - \\
Peso (kg) & 101 & 20,3 & 14,55 & - \\
Talla (cm) & 101 & 110,76 & - & 84,2 \\
ASA & & - & - & 15,8 \\
I & 85 & - & 13,7 & - \\
II & 16 & 44,83 & - & 13,9 \\
Tiempo Qx (min) & 101 & & - & 86,1 \\
Técnica Qx & 14 & - & - & \\
Fría & 87 & & & \\
Caliente & & & & \\
\hline
\end{tabular}

n: número de pacientes; DE: desviación estándar; \%: porcentaje; Tiempo Qx: tiempo quirúrgico; Técnica Qx: técnica quirúrgica. Edad, peso, talla y tiempo Qx expresados, como media \pm desviación estándar. Sexo, estado físico ASA y técnica Qx expresados como frecuencia y porcentaje. 


\section{Tabla 2. Dolor postoperatorio evaluado por The Children's Hospital of Eastern Ontario Pain Scale} (CHEOPS)

\begin{tabular}{lrrr}
\hline CHEOPS & $\mathbf{n}$ & $\%$ & $\mathbf{p}$ \\
$\leq 4$ & 89 & 88,1 & 0,001 \\
$5-10$ & 8 & 7,9 & \\
$\geq 11$ & 4 & 4,0 & \\
Total & 101 & 100,0 & \\
\hline
\end{tabular}

n: número de pacientes.

\begin{tabular}{|c|c|c|c|c|c|c|c|c|c|}
\hline \multirow{2}{*}{$\begin{array}{l}\text { T é c n i c a } \\
\text { Quirúrgica }\end{array}$} & \multicolumn{6}{|c|}{ CHEOPS } & \multicolumn{2}{|c|}{ Total } & \multirow[t]{2}{*}{$p$} \\
\hline & & & & & & & & & \\
\hline Fría & 13 & $(92,86 \%)$ & 0 & $(0,00 \%)$ & 1 & $(7,14 \%)$ & 14 & $(100 \%)$ & 0,001 \\
\hline Caliente & 76 & $(87,36 \%)$ & 8 & $(9,20 \%)$ & 3 & $(3,45 \%)$ & 87 & $(100 \%)$ & 0,015 \\
\hline$p$ & 0,93 & 0,001 & 0,25 & & & & & & \\
\hline
\end{tabular}

Valores expresados como frecuencias y (porcentajes).

(Tabla 2). Los pacientes que requirieron refuerzo analgésico totalizaron 12 pacientes para un 11,9\% de la muestra y sólo ameritaron una dosis de rescate.

Posteriormente, se correlacionaron los pacientes operados con técnica fría y caliente con el grado de dolor que presentaron, evidenciando que de 14 pacientes operados bajo técnica fría 13 presentaron puntuaciones según la CHEOPS $\leq 4$ puntos y $1 \geq 11$ puntos, con una $p=0,001$; de los 87 operados con técnica caliente 76 presentaron puntuaciones según la CHEOPS $\leq 4$ puntos, 8 entre 5 - 10 puntos y $3 \geq 11$ puntos, con diferencia estadísticamente significativa. No hubo diferencia estadísticamente significativa entre los grupos de técnica fría y caliente con relación al puntaje según la CHEOPS (Tabla 3).

El tiempo de recuperación según Aldrete modificado, el tiempo hasta la primera ingesta oral y el primer momento en que los individuos refirieron dolor posterior al alta se relacionó con el grado de dolor, resultando: los pacientes con puntuación según la CHEOPS $\leq 4$ puntos presentaron una media de 50,13 \pm 13,91 min para la recuperación, 3,08 \pm 0,83 horas para la tolerancia oral y $18,3 \pm 7,41$ horas para el momento en que refirieron dolor; en los pacientes con puntuación según la CHEOPS entre 5 - 10 puntos se evidenció un tiempo de 63,75 \pm 10,61 min para la recuperación, $4 \pm 0$ horas para primera ingesta oral y 5,75 $\pm 0,89$ horas para el momento en que se manifestó dolor; y, para los pacientes con puntuación según la CHEOPS $\geq 11$ puntos, el tiempo de recuperación fue de $86,25 \pm 7,5 \mathrm{~min}$, para la primera ingesta oral de $4 \pm 0$ horas y el momento en que refirieron dolor de 4,5 $\pm 1,0$ horas. No hubo diferencia estadísticamente significativa entre los grupos con respecto al tiempo de recuperación y tiempo para la tolerancia oral con $p=0,618$ y 0,828 respectivamente, siendo estadísticamente significativo para el momento en que manifestaron dolor posterior alta con una $p$ de 0,001 entre el grupo de puntuación CHEOPS $\leq 4$ puntos y los restantes (Tabla 4).

No se observaron complicaciones asociadas al bloqueo ni a la cirugía en la muestra estudiada.

\section{Discusión}

Nuestros resultados demostraron que la aplicación del BRL del NGF para ADT en pacientes pediátricos es una excelente opción para el manejo del DPO evaluado por la CHEOPS, con disminución en los tiempos de recuperación y de tolerancia oral, y mayor tiempo de analgesia.

La analgesia IV ha sido el pilar para el manejo del DPO de pacientes intervenidos de ADT a lo largo de los años, donde el uso de AINEs, opioides, dexametasona y antagonistas NMDA han demostrado su 


\begin{tabular}{|c|c|c|c|c|}
\hline & & CHEOPS & & $\mathbf{p}$ \\
\hline & $\leq 4$ & $5-10$ & $\geq 11$ & \\
\hline Tiempo rec (min) & $50,13 \pm 13,91$ & $63,75 \pm 10,61$ & $86,25 \pm 7,5$ & 0,618 \\
\hline Ingesta oral (horas) & $3,08 \pm 0,83$ & $4 \pm 0,00$ & $4 \pm 0,00$ & 0,828 \\
\hline 1er dolor (horas) & $18,30 \pm 7,41$ & $5,75 \pm 0,89$ & $4,5 \pm 1,00$ & 0,001 \\
\hline
\end{tabular}

Tiempo rec: Tiempo de recuperación anestésica según escala de Aldrete modificado, $1^{\text {er }}$ dolor: primer momento en que el paciente manifestó dolor posterior al alta. Valores expresados en medias y desviaciones estándar.

eficacia. Sin embargo, estos presentan una gran variedad de efectos adversos (náuseas, vómitos, estreñimiento, depresión respiratoria, sedación, sangrado, alucinaciones, etc.) que han hecho que el control del dolor sea un verdadero reto para el anestesiólogo y el otorrinolaringólogo, situación en la que el uso de técnicas regionales como la infiltración peritonsilar han demostrado ser de gran ayuda[5].

Un metaanálisis realizado por Sun et al.[6], así como una variedad de estudios prospectivos aleatorizados como los de Peyvandi et al.[7], Shamendra et al.[8] y Basuni[9] et al., nos revelan como el bloqueo peritonsilar es una técnica eficaz para proveer analgesia a pacientes pediátricos sometidos a ADT valorado por escala visual análoga (EVA) y CHEOPS, siendo la más utilizada en la actualidad tanto por otorrinolaringólogos como por anestesiólogos para el manejo del DPO en poblaciones pediátricas.

La infiltración peritonsilar de anestésicos locales puede causar complicaciones como parálisis de las cuerdas vocales, obstrucción severa de la vía aérea superior, edema agudo de pulmón (por bloqueo del vago y el hipogloso), abscesos profundos del cuello y derrame cerebral, que se han visto después de la infiltración profunda y uso de altas dosis de anestésicos locales, por lo que se debe tener precauciones y vigilar al paciente posterior a su uso[10]. Aunado a esto, la infiltración peritonsilar produce alteración de la anatomía donde se va a realizar el procedimiento[8], por lo que puede conllevar a problemas durante la resección como sangrado o aumento del tiempo quirúrgico con disminución del confort del otorrinolaringólogo, por lo que algunos de ellos se niegan a la ejecución de esta técnica como rutina.

Un estudio realizado por Wang et al.[11], nos muestra cómo la rama lingual del NGF provee sensación a la porción posterior de la lengua y la pared faríngea, donde el bloqueo de esta raíz nerviosa puede otorgar condiciones adecuadas para la intubación y el examen físico orofaríngeo con la aplicación de una sola inyección de anestésico a través de la base del pilar anterior admigdalino. Ortega et al.[12], implementaron el uso de este método para mejorar la tolerancia en pacientes para endoscopia digestiva superior, demostrando su eficacia con mínimos requerimientos de sedación en estos individuos. Los datos que apoyan la utilización de esta técnica en ADT son escasos, el metaanálisis realizado por Suresh et al.[13], revela que sólo encontraron un estudio donde se evaluó el uso de anestesia regional (bloqueo glosofaríngeo bilateral) en pacientes pediátricos para mejorar la analgesia para ADT, con disminución en las puntuaciones de dolor postoperatorio medido por EVA en comparación con el grupo control, sin evidencia de complicaciones[14].

Como se observa en nuestros resultados, aproximadamente, el $90 \%$ de los pacientes presentó niveles bajos de dolor medido por la escala validada para evaluación del dolor en poblaciones pediátricas CHEOPS, lo cual demuestra la eficacia analgésica de esta técnica sencilla a un solo abordaje en esta población indistintamente del método quirúrgico utilizado (frío o caliente). Además, el resto de los pacientes, aún cuando presentaron puntuaciones de la CHEOPS superiores, mostraron que un único refuerzo analgésico fue suficiente para el manejo del dolor, por lo que se disminuyó el uso de estos medicamentos.

Con relación a la recuperación, en este estudio se pudo observar que el tiempo para el alta anestésica medida por escala de Aldrete modificado y la primera ingesta oral, fue menor para los pacientes que tuvieron menores puntaciones por la CHEOPS con 50,13 minutos y 3,08 horas respectivamente, pero sin diferencia significativa. Shamendra et al.[8], también evidenciaron que los pacientes a los que se le realizó infiltración peritonsilar con anestésicos locales, tuvieron tiempos de recuperación más cortos con puntajes 8 y 9 puntos según escala de Aldrete a la primera hora 
de haber concluido la intervención y el tiempo para la ingesta oral fue alrededor de 4 horas, datos que asemejan a nuestros resultados. Mohamed et al.[14], evaluaron el tiempo de alta institucional en pacientes que recibían bloqueo del NGF siendo de aproximadamente 19 horas, menor que en aquellos a los que no se les realizaba bloqueo; por lo que esta técnica se asocia a una disminución en los tiempos de recuperación y en la ingesta oral cuando se realiza.

El primer momento en el que los pacientes manifestaron dolor posterior al alta en los individuos que tuvieron valores de CHEOPS $\leq 4$ puntos fue de 18,30 horas aproximadamente, similar a los resultados conseguidos por Shamendra et al.[8], para infiltración peritonsilar con anestésicos locales (20 horas). Por otro lado, los estudios de Mohamed et al.[14] y Basuni et al.[9], evidenciaron tiempos mucho menores a los obtenidos en nuestros resultados, con valores de 7,8 horas con bloqueo de NGF y 6,22 horas con infiltración peritonsilar respectivamente; estos resultados probablemente se deban a que las técnicas se realizaron con anestésicos locales sin la adición de vasoconstrictores, que como es bien sabido, prolongan el tiempo de analgesia de las técnicas regionales[15].

Si comparamos los pacientes que tuvieron menores puntuaciones según la CHEOPS con los que tuvieron mayor puntuación, observamos que hubo una diferencia importante en cuanto al tiempo de analgesia postoperatoria, lo cual pudo deberse a que el bloqueo no se instauró adecuadamente resultando en niveles de dolor superiores y por supuesto tiempo de analgesia menores, sin embargo, esto sólo se observó en el $11,9 \%$ de la muestra estudiada.

A diferencia de la infiltración peritonsilar, el BRL del NGF está asociada a menos complicaciones dentro de la que se describe el riesgo de administración vascular de agentes anestésicos, la cual puede evitarse aspirando previo a la instilación de la mezcla, y obstrucción de la vía aérea superior por bloqueo proximal del nervio vago[16]. En nuestro estudio no observamos complicaciones asociadas a la técnica, incluso el uso de norepinefrina en la mezcla, no sólo permitió alargar el tiempo analgésico de la técnica, sino que colabora a reducir el riesgo de complicaciones como sangrado y la absorción sistémica de anestésicos[17].

Dentro de las limitaciones con las que contó nuestro estudio está que al no ser comparativo no se puede cegar la técnica, lo que crea sesgos en los análisis. Por otro lado, la falta de instrumentos que incrementen la efectividad de la técnica también es otra limitación, como el uso de la ecografía que ya se ha convertido en una herramienta útil para la realización de bloqueos regionales y la cual apenas ha logrado desarrollarse para bloqueo de NGF en adultos[18].

Por ende, para investigaciones futuras, se recomienda realizar estudios comparativos doble ciego con otras técnicas utilizadas para manejo del dolor en este tipo de cirugía como analgesia intravenosa y la infiltración peritonsilar, así como incluir el BRL del NGF en esquemas de analgesia multimodal para control del dolor postoperatorio. Así mismo, la aplicación del bloqueo de NGF ecoguiado sería de gran ayuda para incrementar la eficacia de la técnica.

En conclusión, el BRL del NGF es una técnica útil, sencilla, eficaz y segura, para el manejo del DPO en pacientes pediátricos sometidos a ADT, con disminución en los tiempos de recuperación y la ingesta oral e incrementando el tiempo de analgesia posterior al alta.

\section{Agradecimientos}

Queremos agradecer especialmente a todo el cuerpo docente y de residentes del Servicio de Anestesiología, los cuales siempre estuvieron con nosotros durante el transcurso de la investigación.

Muchas gracias a todas las personas que se involucraron con nosotros y que hicieron posible esto, en particular nuestros padres y familiares que siempre nos acompañaron.

\section{Conflictos de intereses}

Relativo al siguiente manuscrito, existen las siguientes relaciones que podrían ser percibidas como potenciales conflictos de intereses: Dr. Adrián Medina médico anestesiólogo graduado de la Universidad Central de Venezuela, se declara que forma parte del grupo de traducción de títulos de la World Federation of Societies of Anaesthesiologists y desempeña el cargo de Gerente Médico para ABBOTT Laboratories en Paraguay y Bolivia; sin embargo, dichas relaciones no formaron parte en la realización de este estudio. 


\section{Referencias}

1. Cullen KA, Hall MJ, Golosinskiy A. Ambulatory surgery in the United States, 2006. Natl Health Stat Report. 2009 Jan;11(11):125.

2. Ramos SD, Mukerji S, Pine HS. Tonsillectomy and adenoidectomy. Pediatr Clin North Am. 2013 Aug;60(4):793-807.

3. Wong I, St John-Green C, Walker SM. Opioid-sparing effects of perioperative paracetamol and nonsteroidal antiinflammatory drugs (NSAIDs) in children. Paediatr Anaesth. 2013 Jun;23(6):475-95.

4. Barreveld A, Witte J, Chahal $H$, Durieux ME, Strichartz G. Preventive analgesia by local anesthetics: the reduction of postoperative pain by peripheral nerve blocks and intravenous drugs. Anesth Analg. 2013 May; 116(5):1141-61.

5. Hansen J, Shah R, Benzon H. Management of pediatric tonsillectomy pain: a review of the literature. Ambulatory Anesthesia. 2016;3:23-6.

6. Sun J, Wu X, Meng Y, Jin L. Bupivacaine versus normal saline for relief of post-adenotonsillectomy pain in children: a metaanalysis. Int J Pediatr Otorhinolaryngol. 2010 Apr;74(4):369-73.

7. Peyvandi A, Ahmady Roozbahany N, Niknazar S, Hosseinrezai Mahani M. Comparison of peritonsillar infiltration of lidocaine and bupivacaine for manage- ment of postoperative pain of tonsillectomy. 2016;2: 37-42.

8. Shamendra M, Rajkumar J, Vijay M, Ramraj M, Mniram M. Control of post operative pain by pre- tonsillectomy peritonsillar infiltration. Journal of evolution of medical and dental sciences. 2015;4:12052-12067.

9. Basuni AS, Ezz HA, Albirmawy OA. Preoperative peritonsillar infiltration of dexamethasone and levobupivacaine reduces pediatric post-tonsillectomy pain: a double-blind prospective randomized clinical trial. J Anesth. 2013 Dec;27(6):844-9.

10. Ayatollahi $\mathrm{V}$, Behdad S, Hatami M, Moshtaghiun $\mathrm{H}$, Baghianimoghadam B. Comparison of peritonsillar infiltration effects of ketamine and tramadol on post tonsillectomy pain: a doubleblinded randomized placebocontrolled clinical trial. Croat Med J. 2012 Apr;53(2):155-61.

11. Wang C, Kundaria S, Fernandez-Miranda J, Duvvuri U. A description of the anatomy of the glossopharyngeal nerve as encountered in transoral surgery. Laryngoscope. 2016 Sep;126(9):2010-5.

12. Ortega M, Linares B. García M y cols. Glossopharyngeal nerve block versus lidocaine spray to improve tolerance in upper gastrointestinal endoscopy. Gastroenterol Res Pract. 2013;2013:1-4.

13. Suresh S, Schaldenbrand K, WaIlis B, De Oliveira GS Jr. Regional anaesthesia to improve pain outcomes in paediatric surgical patients: a qualitative systematic review of randomized controlled trials. Br J Anaesth. 2014 Sep;113(3):375-90.

14. Mohamed SK, Ibraheem AS, Abdelraheem MG. Preoperative intravenous dexamethasone combined with glossopharyngeal nerve block: role in pediatric postoperative analgesia following tonsillectomy. Eur Arch Otorhinolaryngol. 2009 Nov;266(11):1815-9.

15. Song JH, Shim HY, Lee TJ, Jung JK, Cha YD, Lee DI, et al. Comparison of dexmedetomidine and epinephrine as an adjuvant to $1 \%$ mepivacaine in brachial plexus block. Korean J Anesthesiol. 2014 Apr;66(4):283-9.

16. Garg R, Singhal A, Agrawal K, Agrawal N. Managing endodontic patients with severe gag reflex by glossopharyngeal nerve block technique. J Endod. 2014 Sep;40(9):1498-500.

17. Bameshki AR, Razban M, Khadivi E, Razavi M, Bakhshaee M. The effect of local injection of epinephrine and bupivacaine on posttonsillectomy pain and bleeding. Iran J Otorhinolaryngol. 2013 Sep;25(73):209-14.

18. Ažman J, Stopar Pintaric T, Cvetko E, Vlassakov K. Ultrasoundguided glossopharyngeal nerve block: a cadaver and a volunteer sonoanatomy study. Reg Anesth Pain Med. 2017 Mar/ Apr;42(2):252-8. 